\title{
Efeito dos Jogos Digitais no Comportamento: Análise do General Learning Model
}

\author{
Mauricio Miranda Sarmet ${ }^{1}$ \\ Programa de Pós-Graduação em Psicologia Social, do Trabalho e das Organizações \\ da Universidade de Brasília, Brasília, DF, Brasil \\ Instituto Federal de Educação, Ciência e Tecnologia da Paraíba, João Pessoa, PB, Brasil \\ Ronaldo Pilati \\ Departamento de Psicologia Social e do Trabalho da Universidade de Brasília, \\ Brasília, DF, Brasil
}

\begin{abstract}
Resumo
Os jogos digitais vêm ocupando um espaço maior na vida das pessoas. Com a popularização de computadores pessoais, consoles de videogame e artefatos portáteis, uma quantidade maior de pessoas passa mais tempo exposta a diferentes jogos e diversas áreas do conhecimento estudam quais consequências estão associadas a este fenômeno. Além disso, os jogos digitais são comumente associados a uma série de comportamentos antissociais, como agressões e assassinatos. O objetivo do presente trabalho é discutir um conjunto de evidências sobre a influência de jogos nas dimensões de comportamento, cognição, afeto e excitação, com base no General Learning Model, tendo como foco dados relacionados à agressividade e à prosocialidade. Além disso, busca-se apresentar as principais críticas associadas às pesquisas da área, como a qualidade das medidas utilizadas, a seleção de jogos para pesquisas e o potencial explicativo do modelo. Por fim, sugere-se agenda de pesquisa com o intuito de contribuir com o aprimoramento deste campo de investigação.
\end{abstract}

Palavras-chave: Jogos digitais, General Learning Model, cognição.

\section{The Effect of Digital Games on Behavior: Analysis of the General Learning Model}

\begin{abstract}
Digital games now occupy more space in people's lives. Since the popularization of personal computers, game consoles and portable devices more people spend more time exposed to a greater variety of games. Researchers in various fields have been studying the consequences of this phenomenon. Digital games are commonly associated with a range of antisocial behaviors such as aggression and murder. This paper's objective is to discuss evidence about the influence of games on human behavior, cognition, affect and arousal, based on the General Learning Model, focusing on available data related to aggression and prosociality. Several criticisms of the extant research are discussed: the poor quality of the measures used, the games used in research and the explanatory power of this model. Finally, a research agenda to improve work is this field is proposed.
\end{abstract}

Keywords: Digital games, Geneeral Learning Model, cognition.

Endereço para correspondência: SQSN 313 - D - 602, Brasília, DF, Brasil 70766-040. E-mail: msarmet@ gmail.com e rpilati@gmail.com

Este trabalho recebeu o apoio do Conselho Nacional de Desenvolvimento Científico e Tecnológico (CNPq) e da Coordenação de Aperfeiçoamento de Pessoal de Nível Superior (CAPES).. 


\section{Efecto de los Videojuegos en el Comportamiento: Análisis del General Learning Model}

\section{Resumen}

Juegos digitales han estado ocupando un gran espacio en la vida de las personas. Con la popularización de las computadoras personales, consolas de videojuegos y los artefactos portátiles, una gran cantidad de personas que pasan más tiempo expuestos a diferentes juegos y las diferentes áreas de conocimiento a estudiar qué consecuencias son associadas a este fenómeno. Además, los juegos digitales son comúnmente asociados con una serie de conductas antisociales, como la agresión y el asesinato. El objetivo de este trabajo es discutir una serie de pruebas com respecto a la influencia de los juegos en las dimensiones de la conducta, la cognición, el afecto y la excitación, con base en el General Learning Model, centrándose en los datos relativos a la agresión y prosocialidad. Además, presenta las principales críticas asociadas a la investigación deste objeto, tales como la calidad de las medidas utilizadas, la selección de la investigación de juegos y el poder explicativo del modelo. Por último, se sugiere una agenda de investigación con el fin de contribuir a la mejora de este campo de investigación.

Palabras clave: Juegos digitales, General Learning Model, cognición.

O surgimento de novas mídias traz, inevitavelmente, uma discussão sobre seus efeitos no comportamento humano, seja ele benéfico ou prejudicial. Tal reflexão tem justificativa no fato de que sistemas de comunicação e entretenimento em massa possuem uma capacidade de atingir um grande número de pessoas, com diversos perfis sociais, como no caso do rádio, do cinema e da televisão. No caso dos jogos digitais, tal processo não é diferente. Atualmente, eles fazem parte do cotidiano de pessoas de diversas idades e níveis socioeconômicos, em diferentes formatos (jogos de computador, consoles específicos para este fim, tablets e aparelhos celulares). Cada vez mais, uma quantidade maior de pessoas faz parte de um grupo de consumidores de uma indústria que cresce de maneira vertiginosa a cada dia (Mäyrä, 2008; McGonigal, 2011).

Um dos pontos mais controversos no estudo de jogos digitais está relacionado a casos de assassinatos e outros atos agressivos associados ao consumo de jogos violentos (por exemplo, o caso de Columbine, nos Estados Unidos, e o da escola de Realengo, no Rio de Janeiro). Os argumentos da mídia retratam, de um lado, a barbaridade e a falta de sentido no crime e, do outro, um conjunto de hábitos do assassino relacionados ao consumo de determinados jogos. Fica, de forma implícita ou explícita, a suposição de uma rela- ção causal entre o tipo de jogo que o indivíduo joga e determinados comportamentos que ele manifesta fora do contexto de jogo.

As revisões sistemáticas e meta-análises sobre o tema apresentam, na opinião de seus autores, evidências consistentes o bastante para sustentar a existência de uma relação causal entre a exposição a jogos digitais e determinados comportamentos (por exemplo, Anderson \& Bushman, 2001; Anderson et al., 2010; Barlett, Anderson, \& Swing, 2009; Fischer, Greitemeyer, Kastenmüller, Vogrincic, \& Sauer, 2011; Greitemeyer \& Mügge, 2014). Apesar de existirem críticas com relação a aspectos metodológicos e à relevância prática associadas a estes estudos (Elson \& Ferguson, 2014; Ferguson, 2007, 2009a, 2009b; Ferguson \& Dyck, 2012), alguns autores sugerem que pesquisas futuras não deveriam se debruçar sobre a existência ou não do fenômeno, mas sim sobre os elementos processuais associados a ele (Bushman \& Huesmann, 2014). Para estes autores, o importante agora seria investigar como os jogos podem influenciar o comportamento de seus jogadores, e quais variáveis estariam envolvidas nesta relação. Constata-se, no entanto, escassez nos estudos que buscam avaliar evidências que sustentem todas as premissas que constituem o modelo teórico utilizado, principalmente no que 
se refere ao impacto das características constituintes dos jogos eletrônicos nos processos de avaliação (i.e. automáticos e controlados) dos estímulos recebidos, bem como a influência destes na ocorrência de determinados comportamentos.

Neste sentido, o presente artigo tem como objetivo discutir as evidências empíricas do efeito de jogos sobre o comportamento humano, enfocando principalmente aspectos processuais e metodológicos. Dada a variedade de evidências sobre diferentes aspectos relacionados ao tema, este trabalho se limitará a discutir efeitos principalmente associados a conteúdos agressivos e prosociais. Por fim, pretende-se, com este trabalho, apresentar algumas críticas acerca de decisões metodológicas, como a seleção dos estímulos e medidas utilizados, além de apontar questionamentos sobre o potencial explicativo do modelo teórico comumente utilizado, sugerindo caminhos de investigação, dentro da perspectiva do modelo dual de processamento de informações, para aprimorar o conhecimento sobre o efeito de jogos digitais no comportamento.

Para atingir o objetivo proposto, o presente trabalho apresenta, inicialmente, o modelo teórico mais comumente utilizado em estudos desta natureza. Em seguida, serão apresentadas as evidências acerca da influência de jogos eletrônicos no comportamento humano (contemplando sua influência nas dimensões cognitivas, afetivas e de arousal). Por fim, serão apresentadas as críticas mais comuns a estes modelos e sugestões para pesquisas futuras.

\section{Modelo Geral de Aprendizagem (General Learning Model [GLM])}

O GLM é um modelo que apresenta as principais dimensões envolvidas no estudo da influência de diferentes mídias no comportamento, bem como diferentes rotas pelas quais essa influência parece ocorrer (Buckley \& Anderson, 2006). Este modelo se baseia no General Aggression Model, de Anderson e Bushman (2002), e foi desenvolvido de forma a considerar outros tipos de fenômenos além daqueles relacionados à agressão.
Este modelo propõe, como um dos seus elementos principais, que o impacto de elementos midiáticos ocorre tanto de forma imediata (ou de curto prazo) quanto de longo prazo, em função da exposição repetida a um mesmo estímulo (ou estímulos semelhantes). Neste sentido, algumas características estruturais e processuais dos jogos digitais possibilitam um contexto de aprendizagem que pode gerar mudanças duradouras tanto em disposições quanto em comportamentos (Swing, Gentile, \& Anderson, 2009): (a) a capacidade de controlar e ajustar o nível de dificuldade do processo de aprendizagem, possibilitando que a dificuldade se ajuste ao nível de expertise do jogador; (b) a aplicação repetida de determinadas habilidades, caracterizando um contexto de treino; (c) a existência de um processo ativo de aprendizagem, uma vez que os jogadores devem se engajar intencionalmente na experiência de jogo para cumprir os objetivos propostos; (d) a existência de feedbacks imediatos e de recompensas para as ações dos jogadores; (e) a possibilidade de generalização de comportamentos específicos a contextos diversos, uma vez que habilidades semelhantes são solicitadas em jogos diferentes (por exemplo, em diferentes jogos de FPS - First Person Shooters). Tais características muitas vezes são utilizadas para se justificar o efeito potencializador que o jogo digital possui em comparação com outras mídias, onde a interatividade e o feedback constantes não são frequentes.

Como bases teóricas, o modelo se fundamenta em diversas teorias sobre a cognição e o aprendizado humano: as teorias de aprendizagem social de Bandura, a teoria neo-associacionista de memória de Berkowitz, de transferência de excitação de Zillmann, de Script de Husmann e de processamento social da informação de Crick e Dodge (Barlett \& Anderson, 2013; Buckley \& Anderson, 2006). Neste sentido, os autores defendem que o GLM parte de uma grande quantidade de estudos que, de uma forma ou de outra, fornecem suporte para essas teorias, o que confere maior robustez teórica ao modelo proposto (Prot \& Anderson, 2013).

No GLM (Figura 1) são previstos dois tipos diferentes de inputs. As variáveis pessoais dizem 
respeito a atitudes, crenças, objetivos, experiências prévias, estados emocionais anteriores à exposição ao jogo, entre outros. São consideradas variáveis relativamente estáveis no tempo. As variáveis situacionais, por sua vez, correspondem aos elementos contextuais e envolvem as características da mídia e do contexto, bem como a compreensão que os indivíduos possuem deste contexto específico. Estas variáveis acabam por influenciar o estado interno do indivíduo, composto pelas rotas cognitivas, afetivas e de alerta/ excitação (arousal), que interagem para resultar em um processo de avaliação.

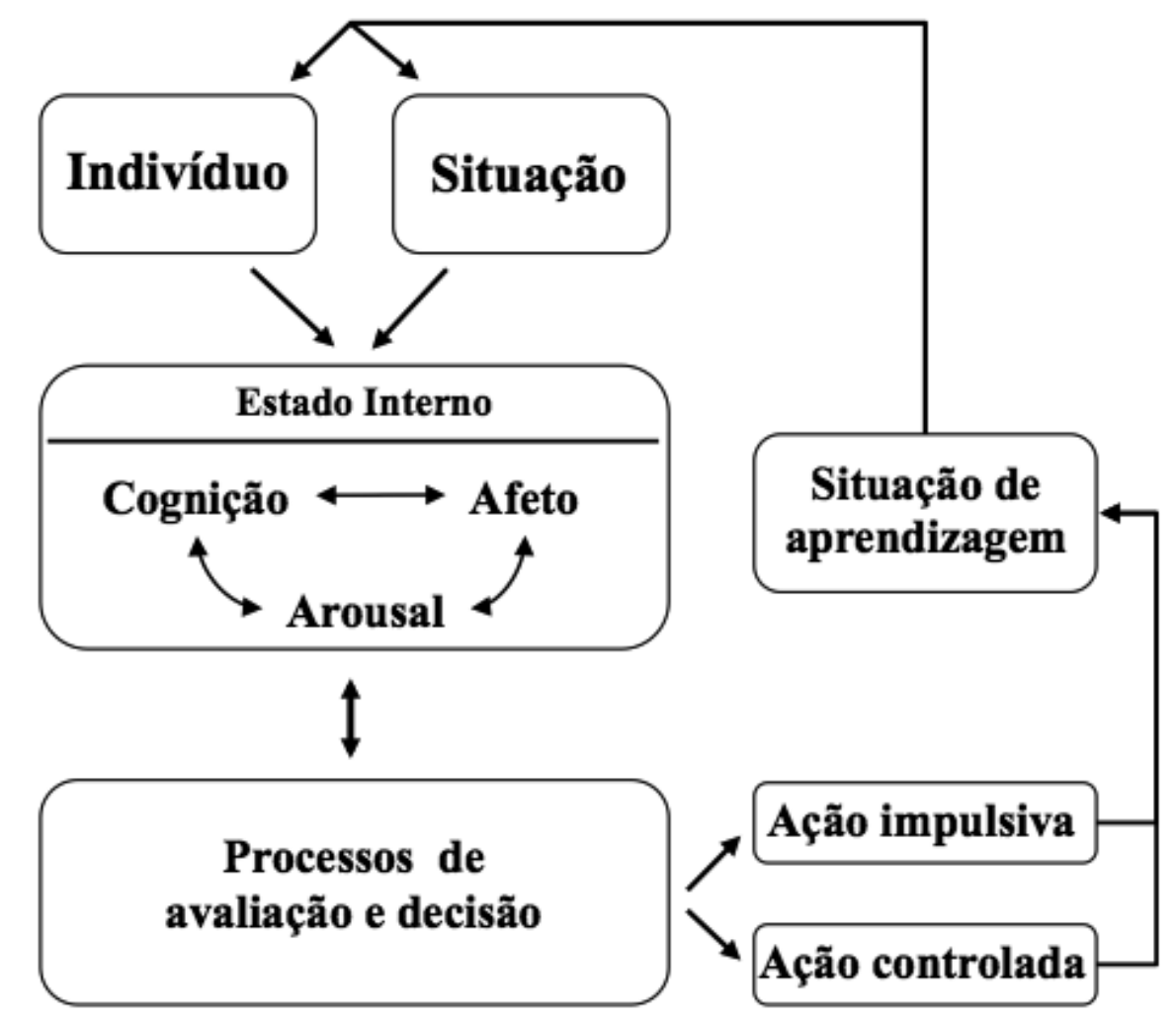

Figura 1. General Learning Model (GLM). Adaptado de Buckley e Anderson (2006).

De acordo com o modelo, determinadas características da situação podem eliciar conteúdos compatíveis em cada uma das rotas do estado interno e, consequentemente, resultar em comportamentos também similares. Neste sentido, o GLM hipotetiza que um jogo violento, por exemplo, ativará conteúdos relacionados à violência da memória, além de estados afetivos e de excitação (arousal) congruentes ao contexto violento, ocasionando em comportamentos mais agressivos. Além disso, pressupõe efeitos de longo prazo que envolvem o aprendizado fatual e de comportamentos (a partir do treino recorrente, reforçamentos e punições), além de mudanças disposicionais (Buckley \& Anderson, 2006). A ocorrência de efeitos de curto e de longo prazo, no entanto, parecem ocorrer em função de processos diferenciados: enquanto os efeitos de curto prazo parecem estar relacionados a efeitos de priming, arousal e de imitação, os efeitos de longo prazo envolvem aprendizagem por observação e ativação e dessensibilização de processos emocionais (Huesmann, 2007).

Em suma, o GLM pressupõe que a interação com os jogos digitais (em função de seu caráter de simulação) possibilita que o conhecimento produzido a partir da experiência de jogo influenciem a percepção e interpretação de situações de jogo, podendo envolver estados afetivos, estruturas cognitivas e scripts comportamentais que guiarão o comportamento dos jogadores em 
situações futuras. A partir do treino recorrente característico da situação de jogo, a ativação destes padrões pode se tornar mais acessível, alterando a forma como o indivíduo interpreta e lida com as situações externas ao jogo.

Um ponto importante do GLM diz respeito ao processamento das informações recebidas. Após a exposição ao estímulo, este processo avaliativo ocorre de forma imediata (Barlett \& Anderson, 2013). A depender da quantidade de recursos atencionais e de quão satisfatório for o resultado desta avaliação, um processo de reavaliação (mais controlado) pode ser ativado (Anderson \& Dill, 2000). A diferença entre processos automáticos ou controlados de avaliação resultam, respectivamente, em ações impulsivas ou controladas, e suscitam uma discussão sobre como as informações recebidas pelo meio, bem como os padrões mnemônicos ativados a partir desta exposição, resultam em determinados padrões comportamentais. Neste sentido, o GLM sugere que a existência de recursos cognitivos, bem como o resultado preliminar da avaliação, podem demandar o uso de estratégias mais controladas de escolha do comportamento a ser manifestado.

Este aspecto, apesar de ser ainda escasso na literatura sobre jogos digitais, mostra-se relevante para se compreender tanto os resultados empíricos quanto a significância prática de tais achados. Isso porque, caso as proposições do modelo sejam coerentes, o comportamento efetivamente observado não depende somente do tipo de jogo digital consumido (nem de sua natureza, por exemplo, violenta ou prosocial); a influência deste sobre o primeiro seria mediada por uma série de outras variáveis, como a capacidade do indivíduo filtrar suas primeiras avaliações de forma a controlar o tipo de comportamento que considera relevante manifestar.

Vários estudos foram realizados na tentativa de investigar a relação entre as características do jogo e sua influência em diferentes dimensões do GLM. Importante notar que, dado o caráter genérico do modelo e a quantidade de variáveis que o compõe, torna-se inviável testá-lo em sua completude em um mesmo estudo. Em função disso, as evidências produzidas em favor do mo- delo contemplam relações entre variáveis específicas. A robustez do modelo, de acordo com seus proponentes, se dá justamente em função de uma análise conjunta das evidências encontradas relacionando as características da mídia com as principais rotas (cognitiva, afetiva e de excitação) e com o comportamento. A seguir, serão apresentadas evidências para cada uma dessas dimensões.

\section{Evidências Empíricas sobre o GLM}

De forma geral, observa-se que é mais comum o uso de delineamentos experimentais para se verificar a influência dos jogos digitais sobre as diversas dimensões do GLM, em função da necessidade de manipulação e de um maior controle de variáveis (Greitemeyer \& Mügge, 2014). Dentro deste escopo, manipula-se a natureza do jogo a ser utilizado (por meio de jogos prosociais, violentos e neutros, por exemplo) dentro de grupos experimentais diferenciados.

Considerando o comportamento como principal variável dependente, os resultados de vários estudos independentes sugerem que a natureza do jogo pode eliciar comportamentos congruentes com ela. Observa-se, por exemplo, que jogar jogos violentos pode aumentar a ocorrência de comportamentos agressivos medidos pelo Competitive Reaction Time Task - CRTT (Anderson \& Dill, 2000; Carnagey \& Anderson, 2005). Nesta tarefa, simula-se uma situação de competição entre jogadores onde é facultado ao vencedor disparar um sinal sonoro de intensidade e duração variáveis e, quanto mais alta é a intensidade (e mais duradoura) escolhida pelo participante, mais agressivo se compreende o seu comportamento. Ainda, a exposição a jogos violentos tendem a reduzir o comportamento de doação (Chambers \& Ascione, 2001). De forma contrária, jogos prosociais estão associados a uma maior intenção em ajudar terceiros (Greitemeyer \& Osswald, 2010; Jin, 2011; Peng, Lee, \& Heeter, 2010), selecionar quebra-cabeças mais fáceis para outro participante (Gentile et al., 2009; Saleem, Anderson, \& Gentile, 2012a) e ajudar ostensivamente um confederado, coletan- 
do lápis que caíram no chão ou auxiliando-o em uma situação simulada de conflito (Greitemeyer \& Osswald, 2010).

De forma semelhante, a exposição a jogos prosociais parecem reduzir a ocorrência de comportamentos agressivos (Greitemeyer, Agthe, Turner, \& Gschwendtner, 2011). No entanto, não há consenso na literatura, e alguns estudos apresentam evidências de que o efeito pode não existir (Ferguson, 2007; Sestir \& Bartholow, 2010; Tear \& Nielsen, 2013; Valadez \& Ferguson, 2012). Além disso, alguns autores criticam o uso do CRTT como medida do comportamento agressivo, argumentando a falta de validade ecológica da medida (Elson \& Ferguson, 2014). As críticas ao uso desta medida contemplam tanto a falta de padronização nas instruções, procedimentos e escalas utilizadas, quanto nas estratégias de análise (Elson, Mohseni, Breuer, Scharkow, \& Quandt, 2014), resultando em um conjunto de inconsistências que fragilizam a medida, podendo inclusive resultar em conclusões diferentes a depender das estratégias de tratamento e análise de dados selecionadas pelo pesquisador.

Ao se considerar a dimensão cognitiva, as principais evidências tratam da ativação, a partir da apresentação de um jogo com características específicas, de determinados conteúdos na memória. De forma geral, essa proposta é operacionalizada a partir da medida de acessibilidade de um conteúdo na memória após a exposição a um jogo com conteúdo semelhante. Por exemplo, Anderson e Dill (2000) expuseram participantes a um jogo violento (Mortal Kombat) ou neutro (Myst); após o período de jogo, solicitaram que os participantes realizassem um teste de velocidade de leitura. Os autores encontraram tempos menores de resposta para a leitura de palavras agressivas em participantes expostos ao jogo violento, e interpretaram tais evidências como um indicador de que conteúdos violentos estariam mais "acessíveis", resultando em um menor tempo de leitura.

Outra medida utilizada é o teste de completamento de palavras (Word Completion Task). Nela, são apresentados aos participantes um conjunto de fragmentos de palavras e é solicitado que eles completem cada um deles com a primeira palavra que vier à mente. Evidências empíricas indicam que a exposição a jogos violentos está associada a uma escolha maior por palavras violentas (Carnagey \& Anderson, 2005; Sestir \& Bartholow, 2010). De forma semelhante, a exposição a jogos prosociais tende a reduzir cognições agressivas (Greitemeyer et al., 2011; Greitemeyer \& Osswald, 2009). Outros estudos utilizaram a tarefa de completar histórias ambíguas, sugerindo que jogos prosociais podem aumentar a acessibilidade a cognições prosociais (e.g., Greitemeyer \& Osswald, 2010).

Ainda considerando evidências sobre a rota cognitiva, estudos relacionaram a exposição a jogos violentos com o autoconceito agressivo implícito, por meio do Teste de Associação Implícita (Bluemke, Friedrich, \& Zumbach, 2009; Uhlmann \& Swanson, 2004). Considerando o caráter ativo característico da experiência de interação, uma vez que o indivíduo comanda o que o personagem faz e é recompensado pelo comportamento agressivo dentro do jogo, os resultados encontrados evidenciam que os indivíduos associam mais conceitos agressivos a si do que a outras pessoas, quando em contato com jogos desta natureza. Outros estudos associam a exposição a jogos digitais com sexualidade, estereotipização de gênero (Yao, Mahood, \& Linz, 2010) e percepção de humanidade (Bastian, Jetten, \& Radke, 2012). Ainda, evidências adicionais sobre o papel da cognição tem apontado, por exemplo, que o efeito pode não ser duradouro (Sestir \& Bartholow, 2010), e que um intervalo de 15 minutos entre a fase de jogo e a medida de cognição resultam em um declínio de cognições agressivas. Há, por fim, críticas à forma como as medidas de cognição são operacionalizadas. Bösche (2010), por exemplo, apresenta evidências de que a exposição a jogos violentos aumenta a acessibilidade tanto de conteúdos agressivos quanto de prosociais. A partir de um teste de decisão lexical, Bösche sugere que as medidas deveriam considerar tanto cognições positivas quanto negativas ao se medir o efeito dos jogos. 
Estudos sobre o impacto dos jogos digitais na dimensão afetiva enfocam, principalmente, a medida de estados afetivos por meio de escalas explícitas, com a Positive and Negative Affect Schedule e a State Hostility Scale, na tentativa de se verificar possíveis alterações destes estados a curto prazo. Sínteses das principais evidências empíricas sobre agressividade (Anderson \& Bushman, 2001; Anderson et al., 2010) e prosocialidade (Greitemeyer \& Mügge, 2014) apontam que a exposição a jogos violentos tendem a aumentar a ocorrência de afetos negativos e reduzir a de afetos positivos, quando comparados a jogos neutros ou prosociais (e uma relação inversa pode ser observada no caso destes últimos). Exemplos de construtos associados a essa dimensão versam sobre, além de estados afetivos, processos de dessensibilização (Huesmann, 2007), cooperação e empatia (Greitemeyer, 2013b), bem como sentimentos agressivos (Jeong, Biocca, \& Bohil, 2012).

No entanto, a relação entre a mudança nos estados afetivos e comportamentos subsequentes parece não estar clara. Por exemplo, em um estudo comparando jogos violentos e neutros, observou-se que, apesar dos grupos expostos ao jogo violento apresentarem índices maiores de afetos negativos, a explicação de comportamentos agressivos parece estar mais associadas à acessibilidade a cognições negativas do que ao afeto (Carnagey \& Anderson, 2005). Resultados similares foram encontrados no estudo da relação entre jogos prosociais (Greitemeyer, 2011). Além disso, alguns estudos não encontraram resultados significativos entre o tipo de jogo e os estados afetivos (por exemplo, Greitemeyer \& Osswald, 2010; Valadez \& Ferguson, 2012), o que sugere que a alteração de estados afetivos poderia ou estar associada a outras características do jogo (que não sua natureza) ou mesmo ao nível de violência ou prosocialidade necessários para que os efeitos ocorram. Em outras palavras, é factível supor que, quanto mais violento o jogo for, mais provável é o efeito sobre o afeto ocorrer, e jogos com níveis superficiais de violência possam não ser suficientes para eliciar tal efeito. Problemas com as medidas explícitas de afeto também são apontadas como possíveis explicações para a inconsistência dos achados na literatura (Elson \& Ferguson, 2014; Saleem, Anderson, \& Gentile, 2012b).

A dimensão de excitação (arousal) é a que possui evidências mais contraditórias. Por um lado, estudos que utilizam medidas fisiológicas para se investigar o impacto de jogos sugerem que o efeito pode existir. Por exemplo, ao estudar o efeito de jogos violentos nos indivíduos, pesquisas evidenciaram mudança significativa na frequência cardíaca, na pressão arterial e no índice de resposta galvânica da pele (Bailey, West, \& Anderson, 2009; Baldaro et al., 2004). Além disso, estudos de revisão sistemática relatam evidências para a relação entre excitação e jogos violentos (Anderson \& Bushman, 2001; Anderson et al., 2010). No caso de jogos prosociais, o único estudo meta-analítico disponível até o momento não encontra resultados significativos (Greitemeyer \& Mügge, 2014). Alguns estudos, no entanto, sugerem que esse efeito pode ocorrer não só em função da natureza do jogo (violento, neutro ou prosocial, por exemplo), mas também em função de elementos gráficos e da imersão que o jogo proporciona ao jogador (Bailey et al., 2009). Há, ainda, estudos que não encontram significância estatística ao se estudar essa dimensão (Jeong et al., 2012).

As evidências apresentadas até o momento elucidam, por um lado, alguns questionamentos sobre a influência dos jogos digitais no comportamento humano e, por outro, apresentam novas questões de pesquisa buscando melhor compreender este fenômeno. Estas novas questões necessitam ser exploradas buscando-se identificar e avaliar os efeitos de outras variáveis que podem exercer efeitos de moderação e mediação entre as variáveis preconizadas no GLM. A próxima seção procura abordar este aspecto necessário no estado atual da produção teórica no tema.

\section{Estudos sobre Variáveis Mediadoras}

Considerando a diversidade de variáveis previstas no GLM, faz-se necessário compreender quais outras variáveis podem intermediar a relação entre jogos e comportamento, o que 
pode inclusive esclarecer os diferentes resultados encontrados na literatura. Para se atingir este objetivo, uma estratégia natural de pesquisa é explorar relações de mediação entre variáveis. Pesquisadores que estudam tanto o comportamento agressivo (Barlett \& Anderson, 2013) quanto prosocial (Greitemeyer \& Osswald, 2009) tem sugerido que se considerar variáveis mediadoras é um dos caminhos mais profícuos para o desenvolvimento teórico e metodológico da área.

Conforme ressaltado anteriormente, uma das primeiras evidências empíricas de mediação está relacionada à dimensão cognitiva, ao se considerar como variável dependente tanto o comportamento agressivo quanto prosocial (Anderson \& Dill, 2000; Carnagey \& Anderson, 2005; Greitemeyer et al., 2011; Greitemeyer \& Osswald, 2010). Isso sugere um papel central da cognição como elemento processual: os conceitos, scripts e esquema ativados pela exposição ao jogo, bem como a forma como as informações são interpretadas, parecem ser fundamentais para a manifestação de comportamentos coerentes (ou destoantes) do conteúdo exposto. Coerente com este argumento, outros mediadores relacionados à cognição acabam por ter suporte empírico.

No caso de jogos violentos, um mediador é o viés de expectativa hostil (que envolve a tendência de perceber, como o nome sugere, uma intenção hostil no comportamento de terceiros). Por exemplo, estudos investigaram o efeito da natureza do jogo (violento ou não violento) no comportamento agressivo, e mensuraram o viés de expectativa hostil por meio da tarefa de completar histórias ambíguas. Nesta tarefa, era solicitado aos participantes que listassem o que o protagonista estava pensando, sentindo e fazendo no decorrer da história (Hasan, Bègue, \& Bushman, 2012; Hasan, Bègue, Scharkow, \& Bushman, 2013). As análises sugeriram que a exposição ao jogo violento impactou em interpretações mais hostis das histórias, e que quanto mais hostil era a interpretação, maior era a ocorrência de comportamentos violentos (medidos por meio do Competitive Reaction Time Task). Outras variáveis mediadoras encontradas rela- cionadas à forma como os indivíduos interpretam a situação são a percepção de agressividade (Greitemeyer, 2013a) e o viés intergrupo (Greitemeyer, 2013c).

Ainda sobre jogos violentos, outro mediador relevante é a presença, ou seja, a capacidade de imersão que o jogo pode proporcionar ao jogador (Jeong et al., 2012). Neste estudo, observou-se que o nível de realismo do jogo exerce influência sobre a agressividade dos jogadores, mas somente quando há uma imersão significativa. É importante considerar que este efeito ocorre independente de outras características do jogo, como a perspectiva (primeira ou terceira pessoa) ou a excitação.

No caso de comportamentos prosociais, uma das principais variáveis mediadoras encontradas é a empatia, cujas evidências empíricas foram encontradas inclusive em estudos longitudinais e transculturais (por exemplo, Prot et al., 2013). Mais especificamente, quanto mais o jogo elicia sentimentos de preocupação com o outro, mais frequentes podem ser os comportamentos de ajuda, mesmo em outros contextos. Algumas características de jogos, como a necessidade de cooperação entre jogadores (ou personagens controlados pelo computador) podem aumentar a chance de tal relação empática ocorrer (Greitemeyer, 2013b).

As evidências apresentadas, bem como as críticas associadas a elas, levantam diversos questionamentos que devem ser respondidos, caso se busque uma melhor compreensão deste fenômeno. Neste sentido, alguns pontos de reflexão são apresentados a seguir, bem como sugestões para a realização de estudos futuros.

\section{Aprofundando o Estudo de Jogos: Investigação de Processos Psicológicos e Desenvolvimento de Ferramentas de Pesquisa}

Conforme abordado no início deste trabalho, há um número considerável de evidências que dão suporte à hipótese de que a exposição a jogo digital de uma determinada natureza influencia a forma como os indivíduos interpretam 
diferentes situações dentro e fora do jogo, bem como a forma como se comportam em outros contextos. Ao longo do texto, foram apresentados resultados de algumas pesquisas experimentais que dão suporte a tal afirmação, com o propósito de ilustrar o estado atual da pesquisa nessa temática. No entanto, várias lacunas ainda merecem atenção com vistas a esclarecer pontos ainda não resolvidos, como a dificuldade na replicação de efeitos relevantes, da importância de outras variáveis (como variáveis processuais e disposicionais) para a compreensão do fenômeno, problemas relacionados à validade externa $\mathrm{e}$ à pequena magnitude dos efeitos encontrados, além da fragilidade das medidas utilizadas (E1son \& Ferguson, 2014; Ferguson, 2009a, 2009b; Ferguson \& Kilburn, 2010). O aprofundamento da compreensão do fenômeno, neste sentido, é fundamental para que se possa verificar o nível de adequação do GLM, dado o seu caráter genérico, como modelo explicativo.

Com o intuito de contribuir para o esclarecimento de tais pontos, são apresentadas sugestões para o refinamento de pesquisas experimentais sobre a temática considerando duas vertentes: um critério maior para a seleção (ou desenvolvimento) de jogos a serem utilizados em delineamentos experimentais e a investigação da influência de processos automáticos e controlados na avaliação, julgamento e comportamento do jogador.

\section{Considerações sobre a Seleção dos Jogos Digitais}

A decisão sobre quais jogos utilizar em delineamentos experimentais deve levar em conta diferentes elementos. Um deles, mais aparente, diz respeito à natureza (ou conteúdo) do jogo. Neste sentido, analisa-se quais conteúdos se deseja eliciar a partir da exposição do participante ao jogo, e quais são as variáveis dependentes de interesse. A seleção de jogos caracterizados como violentos, neutros ou prosociais é um exemplo. No entanto, os jogos possuem diversas características além de seu conteúdo, como mecânica de jogo, narrativa, elementos gráficos e sonoros, periféricos de entrada e comandos e complexi- dade, vários deles influenciando as principais variáveis do GLM (Barlett et al., 2009, para uma síntese). Considerando a necessidade de controle para a realização de delineamentos experimentais, bem como o fato de que as características do jogo são elementos situacionais que, de acordo com o GLM, podem influenciar os estados internos dos indivíduos, duas opções podem ser utilizadas: selecionar jogos comerciais (jogos produzidos por empresas, comercializado para o público comum) ou investir no desenvolvimento de jogos especialmente planejados para a pesquisa (McMahan, Ragan, Leal, Beaton, \& Bowman, 2011).

A utilização de jogos comerciais possui, como principais vantagens: disponibilidade imediata e maior validade ecológica. No entanto, os pesquisadores devem garantir, seja por procedimentos de pré-teste ou checagem de manipulação, que o jogo possui as características relevantes para a manipulação da variável independente dentro das condições experimentais e que outras variáveis não estão a comprometer a análise dos resultados. Comumente, a estratégia de inserir questões que versem sobre a percepção de dificuldade, excitação, agradabilidade, satisfação, entre outros, é utilizada em procedimentos de análise post facto. No entanto, alguns autores colocam em dúvida se essa estratégia é suficientemente robusta para excluir as variáveis intervenientes como possíveis explicações para os resultados obtidos (Elson \& Ferguson, 2014).

Por outro lado, o desenvolvimento de jogos digitais específicos, especialmente ao se considerar o fenômeno de interesse deste trabalho, é uma estratégia pouco utilizada pela literatura e mais afinada com as atuais necessidades metodológicas para o desenvolvimento teórico do campo. Jogos personalizados possuem a vantagem de poderem ser detalhadamente construídos para que o controle e a manipulação das variáveis de interesse sejam feitos de forma rigorosa, aumentando a qualidade das evidências encontradas. $\mathrm{O}$ processo de desenvolvimento de um jogo semelhante aos jogos comercializados requer o envolvimento de profissionais especializados, demandando recursos nem sempre dis- 
poníveis pelos grupos de pesquisa, sem ignorar que geralmente é um processo que requer tempo (McMahan et al., 2011). Com isso, tentativas neste sentido podem resultar em produtos que se distanciam muito do que os participantes veriam como um jogo "de verdade". Apesar desta característica, entende-se que o desenvolvimento de jogos específicos traz contribuições mais eficientes para a produção de delineamentos metodológicos mais robustos e consequente compreensão mais refinada dos processos cognitivos, afetivos e comportamentais consequentes ao envolvimento com o jogo eletrônico. Desta feita considera-se que é fundamental o investimento no desenvolvimento de jogos dedicados ao teste de hipóteses específicas sobre os processos envolvidos, de forma a suprir esta lacuna que impede o desenvolvimento adequada da teoria robusta que permita uma melhor compreensão dos efeitos relatados na área de investigação.

\section{Investigação de Processos Automáticos e Controlados}

As evidências empíricas apresentadas neste trabalho acerca da dimensão cognitiva reforçam sua relevância para a compreensão da influência de jogos digitais no comportamento. No GLM, os autores pressupõem a existência de processos diferenciados de avaliação e reavaliação, ativados em função da existência de recursos cognitivos suficientes e de resultados satisfatórios, ocasionando em ações ora impulsiva, ora controladas. No entanto, não apresentam evidências empíricas suficientes para justificar a inclusão de tais elementos no modelo, nem para compreender como os outros elementos do modelo (principalmente, as características do jogo e os elementos contextuais durante a experiência de jogo) interagem com tais processos. Esta é uma lacuna basilar na pesquisa, que coloca em cheque várias das pressuposições do próprio GLM.

A visão de um sistema duplo de processamento de informações não é exclusivo da área de estudo de jogos digitais. A ideia de que existem processos, não acessíveis à consciência, que influenciam a forma como os indivíduos percebem, interpretam e agem sobre o mundo vem sendo objeto de discussão em várias áreas do conhecimento (Hassin, Uleman, \& Bargh, 2005) e pode-se considerar como a modelagem teórica predominante, atualmente, para a compreensão da cognição humana. Tal modelo tem sido utilizado no estudo de processos associados a raciocínio, julgamento e processos decisórios, percepção social, estereótipos, mudança de atitude, entre outros (Evans, 2008), e é embasado por um conjunto de evidências que sugere que o comportamento humano é regido por uma série de processos que não necessariamente ocorrem em um nível explícito de consciência (Dijksterhuis \& Aarts, 2010).

A discussão sobre processamento implícito - o conjunto de processamento de informações que ocorre sem a atenção e/ou controle do indivíduo - é uma questão que vem, há muitos anos, ganhando campo na área de cognição social em função, principalmente, das evidências encontradas em favor do modelo de processamento duplo de informação (Fiske \& Taylor, 2008; Kahneman, 2011). De acordo com este modelo, o processamento cognitivo possui gradações, desde um processamento puramente automático da informação, sem a consciência da presença do estímulo e sem controle do processamento, até um processamento controlado e consciente. Neste sentido, o processamento implícito de informações recebidas do ambiente influenciam de forma sistemática processos decisórios em diversos contextos (Bargh, Schwader, Hailey, Dyer, \& Boothby, 2012).

Dentro desta perspectiva, são escassos os estudos que busquem verificar, empiricamente, o efeito de tais processos na compreensão da influência de jogos no comportamento. As evidências encontradas dizem respeito da influência do conteúdo do jogo no autoconceito agressivo implícito (Bluemke et al., 2009; Uhlmann \& Swanson, 2004). No entanto, outros questionamentos podem ser feitos: é coerente supor que, na ausência de recursos cognitivos, as vias automáticas de processamento de informações tenham um papel mais influente, uma vez que é mais difícil para o indivíduo exercer o controle sobre cada etapa de interpretação e julgamento das informações. Nestes casos, questiona-se quais seriam 
os resultados esperados a partir da exposição a tipos diferentes de jogos, e o papel de variáveis individuais e situacionais. Uma hipótese factível, por exemplo, seria a de que características disposicionais (como agressividade, empatia e prosocialidade) possam ser mais relevantes para predizer a ocorrência do comportamento do que o tipo de jogo, em situação de escassez de recursos. Essa, e outras possíveis hipóteses, requerem uma linha de pesquisa que parece ter sido ignorada pela área até o momento. Neste sentido, sugere-se que novas perspectivas de pesquisa venham a contemplar, por meio de delineamentos experimentais, o efeito da escassez de recursos, de interferência, bem como de abordagens que considerem o modelo duplo de processamento, na relação entre jogos digitais e comportamento, considerando a dimensão cognitiva não somente como uma variável dependente do modelo, mas também como uma variável independente ou mediadora.

Exemplos de tais manipulações geralmente envolvem uma tarefa simultânea de memorização de dígitos. Como os trabalhos de Cavallo, Holmes, Fitzsimons, Murray e Wood (2012) e de Conway e Gawronski (2013), ou uma tarefa de ego depletion (Fennis, 2011; Xu, Bègue, \& Bushman, 2012).

\section{Considerações Finais}

Nos últimos anos, os estudos sobre a influência de jogos digitais têm crescido de maneira significativa. A quantidade de estudos empíricos realizados resultou na criação de modelos teóricos que buscassem a explicação deste fenômeno, e o General Learning Model pode ser considerado o mais influente deles. Com base em seus pressupostos, diversas variáveis relevantes foram sistematicamente levantadas, buscando investigar a influência de jogos digitais em diferentes dimensões do funcionamento humano, como comportamento, cognição, afeto e excitação (Anderson \& Bushman, 2001; Anderson et al., 2010; Barlett et al., 2009; Greitemeyer \& Mügge, 2014). Apesar disso, várias críticas a aspectos teóricos e metodológicos foram feitas, principalmente relacionadas à qualidade das me- didas utilizadas, à seleção de jogos digitais e até mesmo à existência ou relevância do efeito em si (Elson \& Ferguson, 2014; Ferguson, 2009b). Mais do que isso, sugere-se que a própria área de pesquisa pode estar sendo influenciada por vieses que dão importância a um efeito que, caso exista, não possui relevância prática (Ferguson, 2007, 2008). O presente trabalho, portanto, não tem como finalidade desconsiderar as evidências empíricas produzidas em favor no modelo teórico em questão, nem invalidar totalmente seus prossupostos. Busca, com base nos estudos citados, apontar caminhos que possibilitem o aprimoramento da compreensão do fenômeno a partir de uma análise crítica dos pontos ainda não resolvidos no GLM. Com isso espera-se, parafraseando as palavras de Krahé (2014), fomentar uma postura de fair play nesta área de investigação, levando em conta tanto evidências favoráveis quanto desfavoráveis do modelo em questão.

O presente trabalhou buscou apresentar, de forma concisa, tanto evidências a favor da existência do fenômeno quanto as críticas mais comuns associadas a cada uma delas. Longe de ser considerado um levantamento exaustivo da literatura, teve como finalidade incitar uma discussão profícua sobre as lacunas de conhecimento presentes no campo, indicando caminhos e alternativas para sanar tais lacunas. Obviamente a revisão aqui apresentada não foi exaustiva, considerando que existem várias outras ações necessárias para o aprimoramento da área, como a utilização mais frequente de medidas diretas relacionadas às dimensões de excitação e afeto (como medidas fisiológicas) e a realização de estudos longitudinais, com o intuito de verificar o caráter potencializador da exposição contínua a jogos digitais. No entanto, considera-se que a investigação de elementos associados a uma perspectiva dual de processamento da informação, bem como o esclarecimento mais detido no papel do tipo de jogo, parecem ser caminhos promissores de pesquisa, possibilitando a criação de uma agenda de pesquisa positiva para o desenvolvimento de jogos digitais para uso em delineamentos experimentais, o que permitirá a produção teórica incrementada no campo. 


\section{Referências}

Anderson, C. A., \& Bushman, B. J. (2001). Effects of violent video games on aggressive behavior, aggressive cognition, aggressive affect, physiological arousal, and prosocial behavior: A meta-analytic review of the scientific literature. Psychological Science, 12(5), 353-359. Retrieved from http://www.ncbi.nlm.nih.gov/pubmed/11554666

Anderson, C. A., \& Bushman, B. J. (2002). Human aggression. Annual Review of Psychology, 53, 27-51.

Anderson, C. A., \& Dill, K. (2000). Video Game Questionnaire. Journal of Personality, 6.

Anderson, C. A., \& Dill, K. E. (2000). Video games and aggressivethoughts, feelings, and behavior in the laboratory and in life. Journal of Personality and Social Psychology, 78(4), 772-790.

doi:10.1037//O022-3514.78.4.772

Anderson, C. A., Shibuya, A., Ihori, N., Swing, E. L., Bushman, B. J., Sakamoto, A., ...Saleem, M. (2010). Violent video game effects on aggression, empathy, and prosocial behavior in eastern and western countries: A meta-analytic review. Psychological Bulletin, 136(2), 151173. doi:10.1037/a0018251

Bailey, K., West, R., \& Anderson, C. A. (2009). The influence of video games on social, cognitive, and affective information processing. In $\mathrm{J}$. Decety \& J. T. Cacioppo (Eds.), Handbook of social neuroscience (pp. 1-30). Oxford, UK: Oxford University Press.

Baldaro, B., Tuozzi, G., Codispoti, M., Montebarocci, O., Barbagli, F., Trombini, E., \& Rossi, N. (2004). Aggressive and non-violent videogames: Short-term psychological and cardiovascular effects on habitual players. Stress and Health, 20, 203-208. doi:10.1002/smi.1015

Bargh, J. A., Schwader, K. L., Hailey, S. E., Dyer, R. L., \& Boothby, E. J. (2012). Automaticity in social-cognitive processes. Trends in Cognitive Sciences, 16(12), 593-605. doi:10.1016/j. tics.2012.10.002

Barlett, C. P., \& Anderson, C. A. (2013). Examining media effects: The general aggression and general learning models. In E. Scharrer (Ed.), The International Encyclopedia of Media Studies (Vol. 5, pp. 108-127). Hoboken, NJ: Blackwell.
Barlett, C. P., Anderson, C. A., \& Swing, E. L. (2009). Video game effects--Confirmed, suspected, and speculative: A review of the evidence. Simulation \& Gaming, 40(3), 377-403. doi: $10.1177 / 1046878108327539$

Bastian, B., Jetten, J., \& Radke, H. R. M. (2012). Cyber-dehumanization: Violent video game play diminishes our humanity. Journal of Experimental Social Psychology, 48, 486-491. doi:10.1016/j.jesp.2011.10.009

Bluemke, M., Friedrich, M., \& Zumbach, J. (2009). The influence of violent and nonviolent computer games on implicit measures of aggressiveness. Aggressive Behavior, 35, 1-13. doi:10.1002/ab.20329

Bösche, W. (2010). Violent video games prime both aggressive and positive cognitions. Journal of Media Psychology: Theories, Methods, and Applications, 22(4), 139-146. doi:10.1027/18641105/a000019

Buckley, K. E., \& Anderson, C. A. (2006). A theoretical model of the effects and consequences of playing video games. In P. Vorderer \& J. Bryant (Eds.), Playing video games - Motives, responses, and consequences (pp. 363-378). Mahwah, NJ: LEA.

Bushman, B. J., \& Huesmann, L. R. (2014). Twentyfive years of research on violence in digital games and aggression revisited. European Psychologist, 19(1), 47-55. doi:10.1027/10169040/a000164

Carnagey, N. L., \& Anderson, C. A. (2005). The effects of reward and punishment in violent video games on aggressive affect, cognition, and behavior. Psychological Science, 16(11), 882889. doi:10.1111/j.1467-9280.2005.01632.x

Cavallo, J. V., Holmes, J. G., Fitzsimons, G. M., Murray, S. L., \& Wood, J. V. (2012). Managing motivational conflict: How self-esteem and executive resources influence self-regulatory responses to risk. Journal of Personality and Social Psychology, 103(3), 430-451. doi:10.1037/ a0028821

Chambers, J. H., \& Ascione, F. R. (2001). The effects of prosocial and aggressive videogames on children's donating and helping. Journal of Genetic Psychology, 148(4), 499-505.

Conway, P., \& Gawronski, B. (2013). Deontological and utilitarian inclinations in moral decision making: A process dissociation approach. 
Journal of Personality and Social Psychology, 104(2), 216-235. doi:10.1037/a0031021

Dijksterhuis, A., \& Aarts, H. (2010). Goals, attention, and (un)consciousness. Annual Review of Psychology, 61, 467-490. doi:10.1146/annurev. psych.093008.100445

Elson, M., \& Ferguson, C. J. (2014). Twenty-five years of research on violence in digital games and aggression. European Psychologist, 19(1), 33-46. doi:10.1027/1016-9040/a000147

Elson, M., Mohseni, M. R., Breuer, J., Scharkow, M., \& Quandt, T. (2014). Press CRTT to measure aggressive behavior: The unstandardized use of the competitive reaction time task in aggression research. Psychological Assessment, 26(2), 419432. doi:10.1037/a0035569

Evans, J. S. B. T. (2008). Dual-processing accounts of reasoning, judgment, and social cognition. Annual Review of Psychology, 59, 255-278. doi:10.1146/annurev.psych.59.103006.093629

Fennis, B. M. (2011, June). Can't get over me : Ego depletion attenuates prosocial effects of perspective taking. European Journal of Social Psychology, 585, 580-585. doi:10.1002/ejsp.828

Ferguson, C. J. (2007). Evidence for publication bias in video game violence effects literature: A meta-analytic review. Aggression and Violent Behavior, 12, 470-482. doi:10.1016/j. avb.2007.01.001

Ferguson, C. J. (2008). The school shooting/violent video game link: Causal relationship or moral panic? Journal of Investigative Psychology and Offender Profiling, 5(1-2), 25-37. doi:10.1002/ jip.76

Ferguson, C. J. (2009a). Is psychological research really as good as medical research? Effect size comparisons between psychology and medicine. Review of General Psychology, 13(2), 130-136.

Ferguson, C. J. (2009b). Media violence effects: Confirmed truth or just another X-File? Journal of Forensic Psychology Practice, 9(2), 103-126. doi:10.1080/15228930802572059

Ferguson, C. J., \& Dyck, D. (2012). Paradigm change in aggression research: The time has come to retire the General Aggression Model. Aggression and Violent Behavior, 17(3), 220-228. doi:10.1016/j.avb.2012.02.007

Ferguson, C. J., \& Kilburn, J. (2010). Much ado about nothing: The misestimation and overinter- pretation of violent video game effects in eastern and western nations: Comment on Anderson et al. (2010). Psychological Bulletin, 136(2), 174178; discussion 182-7. doi:10.1037/a0018566

Fischer, P., Greitemeyer, T., Kastenmüller, A., Vogrincic, C., \& Sauer, A. (2011). The effects of risk-glorifying media exposure on risk-positive cognitions, emotions, and behaviors: A meta-analytic review. Psychological Bulletin, 137(3), 367-390. doi:10.1037/a0022267

Fiske, S. T., \& Taylor, S. E. (2008). Dual modes in social cognition. In S. T. Fiske \& S. E. Taylor (Eds.), Social cognition: From brains to culture (pp. 25-50). New York: McGraw-Hill Higher Education.

Gentile, D. A., Anderson, C. A., Yukawa, S., Ihori, N., Saleem, M., Ming, L. K., ...Sakamoto, A. (2009). The effects of prosocial video games on prosocial behaviors: International evidence from correlational, longitudinal, and experimental studies. Personality \& Social Psychology Bulletin, 35(6), 752-763. doi:10.1177/0146167209333045

Greitemeyer, T. (2011). Effects of prosocial media on social behavior: When and why does media exposure affect helping and aggression? Current Directions in Psychological Science, 20(4), 251255. doi:10.1177/0963721411415229

Greitemeyer, T. (2013a). Intense acts of violence during video game play make daily life aggression appear innocuous: A new mechanism why violent video games increase aggression. Journal of Experimental Social Psychology. doi:10.1016/j. jesp.2013.09.004

Greitemeyer, T. (2013b). Playing video games cooperatively increases empathic concern. Social Psychology, 44(6), 408-413. doi:10.1027/18649335/a000154

Greitemeyer, T. (2013c). Playing violent video games increase intergroup bias. Personality and Social Psychology Bulletin, 1-9. doi:10.1177/0146167213505872

Greitemeyer, T., Agthe, M., Turner, R., \& Gschwendtner, C. (2011). Acting prosocially reduces retaliation: Effects of prosocial video games on aggressive behavior. European Journal of Social Psychology, 235-242. doi:10.1002/ ejsp. 1837

Greitemeyer, T., \& Mügge, D. O. (2014). Video games do affect social outcomes: A meta-analytic review of the effects of violent and prosocial video game 
play. Personality \& Social Psychology Bulletin, 1-12. doi:10.1177/0146167213520459

Greitemeyer, T., \& Osswald, S. (2009). Prosocial video games reduce aggressive cognitions. Journal of Experimental Social Psychology, 45, 896900. doi:10.1016/j.jesp.2009.04.005

Greitemeyer, T., \& Osswald, S. (2010). Effects of prosocial video games on prosocial behavior. Journal of Personality and Social Psychology, 98(2), 211-221. doi:10.1037/a0016997

Hasan, Y., Bègue, L., \& Bushman, B. J. (2012). Viewing the world through "blood-red tinted glasses": The hostile expectation bias mediates the link between violent video game exposure and aggression. Journal of Experimental Social Psychology, 48, 953-956. doi:10.1016/j. jesp.2011.12.019

Hasan, Y., Bègue, L., Scharkow, M., \& Bushman, B. J. (2013). The more you play, the more aggressive you become: A long-term experimental study of cumulative violent video game effects on hostile expectations and aggressive behavior. Journal of Experimental Social Psychology, 49(2), 224-227. doi:10.1016/j.jesp.2012.10.016

Hassin, R. R., Uleman, J. S., \& Bargh, J. A. (Eds.). (2005). The new unconscious. New York: Oxford University Press. doi:10.1093/acprof:o so/9780195307696.001.0001

Huesmann, L. R. (2007). The impact of electronic media violence: Scientific theory and research. The Journal of Adolescent Health, 41(Suppl.), S6-S13. doi:10.1016/j.jadohealth.2007.09.005

Jeong, E. J., Biocca, F. A., \& Bohil, C. J. (2012). Sensory realism and mediated aggression in video games. Computers in Human Behavior, 28(5), 1840-1848. doi:10.1016/j.chb.2012.05.002

Jin, S.-A. A. (2011). "My avatar behaves well and this feels right": Ideal and ought selves in video gaming. Social Behavior and Personality, 39(9), 1175-1182.

Kahneman, D. (2011). Thinking fast and slow. New York: Farrar, Straus and Giroux.

Krahé, B. (2014). Restoring the spirit of fair play in the debate about violent video games. European Psychologist, 19(1), 56-59. doi:10.1027/10169040/a000165

Mäyrä, F. (2008). An introduction to game studies. Thousand Oaks, CA: Sage.
McGonigal, J. (2011). Reality is broken. Why games makes us better and how they can change the world. New York: The Penguin Press.

McMahan, R. P., Ragan, E. D., Leal, A., Beaton, R. J., \& Bowman, D. A. (2011). Considerations for the use of commercial video games in controlled experiments. Entertainment Computing, 2(1), 3-9. doi:10.1016/j.entcom.2011.03.002

Peng, W., Lee, M., \& Heeter, C. (2010). The effects of a serious game on role-taking and willingness to help. Journal of Communication, 60, 723742. doi:10.1111/j.1460-2466.2010.01511.x

Prot, S., \& Anderson, C. A. (2013). Research methods, design, and statistics in media psychology. In K. Dill (Ed.), The Oxford handbook of media psychology (pp. 109-136). New York: Oxford University Press.

Prot, S., Gentile, D. A., Anderson, C. A., Suzuki, K., Swing, E., Lim, K. M., ...Lam, B. C. P. (2013). Long-term relations among prosocial-media use, empathy, and prosocial behavior. Psychological Science. doi:10.1177/0956797613503854

Saleem, M., Anderson, C. A., \& Gentile, D. A. (2012a, January). Effects of prosocial, neutral, and violent video games on college students' affect. Aggressive Behavior, 38, 263-271. doi:10.1002/ab.21427

Saleem, M., Anderson, C. A., \& Gentile, D. A. (2012b). Effects of prosocial, neutral, and violent video games on children's helpful and hurtful behaviors. Aggressive Behavior, 38(4), 281-287. doi:10.1002/ab.21428

Sestir, M. A., \& Bartholow, B. D. (2010). Violent and nonviolent video games produce opposing effects on aggressive and prosocial outcomes. Journal of Experimental Social Psychology, 46, 934-942. doi:10.1016/j.jesp.2010.06.005

Swing, E. L., Gentile, D. A., \& Anderson, C. A. (2009). Learning processes and violent video games. In R. E. Ferdig (Ed.), Handbook of research on effective electronic gaming in education (Vol. 2, pp. 876-892). New York: Information Science Reference.

Tear, M. J., \& Nielsen, M. (2013). Failure to demonstrate that playing violent video games diminishes prosocial behavior. PLoS ONE, 8(7), e68382. doi:10.1371/journal.pone.0068382

Uhlmann, E., \& Swanson, J. (2004). Exposure to violent video games increases automatic aggressi- 
veness. Journal of Adolescence, 27(1), 41-52. doi:10.1016/j.adolescence.2003.10.004

Valadez, J. J., \& Ferguson, C. J. (2012). Just a game after all: Violent video game exposure and time spent playing effects on hostile feelings, depression, and visuospatial cognition. Computers in Human Behavior, 28, 608-616. doi:10.1016/j. chb.2011.11.006

Xu, H., Bègue, L., \& Bushman, B. J. (2012). Too fatigued to care: Ego depletion, guilt, and prosocial behavior. Journal of Experimental Social Psychology, 48(5), 1183-1186. doi:10.1016/j. jesp.2012.03.007
Yao, M. Z., Mahood, C., \& Linz, D. (2010). Sexual priming, gender stereotyping, and likelihood to sexually harass: Examining the cognitive effects of playing a sexually-explicit video game. Sex Roles, 62(1-2), 77-88. doi:10.1007/s11199-0099695-4
Recebido: 04/08/2014

$1^{a}$ revisão: $29 / 12 / 2014$

Aceite final: 12/01/2015 